\title{
The separability of space and time: Dimensional interaction in the memory trace
}

\author{
ADDIE DUTTA and JAMES S. NAIRNE \\ Purdue University, West Lafayette, Indiana
}

\begin{abstract}
The speeded-classification paradigm, adapted from the study of perceptual interactions, was used to examine interactions among features of the short-term memory trace. In each of four experiments, a trial began with the presentation of a pair of stimuli, each member of which occurred either first or second in time and above or below a fixation point. One of the two stimuli was then presented again, and the task was to classify its prior temporal (Experiments 1 and 3) or spatial (Experiments 2 and 4) position. The main question of interest asked whether subjects could selectively attend to one of these occurrence dimensions while ignoring irrelevant variation along the other. The results suggested that whereas subjects can selectively ignore temporal or spatial variation when no recall of the irrelevant dimension's value is required, they suffer interference when values on both dimensions must be remembered. The obtained patterns of interference are consistent with postperceptual interactions of the spatial and temporal components of the memory trace.
\end{abstract}

Memory traces presumably contain multiple features of presented events. What aspects of the environment are remembered, and how they are represented, has been the focus of considerable research (e.g., Bower, 1967; Tulving \& Bower, 1974). It is reasonably clear that the features represented in a memory trace depend on both the nature of the to-be-remembered stimulus and the conditions imposed by the task (Tulving \& Watkins, 1975), but many important issues remain unresolved. For example, are memories of events better characterized as integrated, all-or-none fragments (Jones, 1976) or as lists of attributes or features (e.g., Bower, 1967; Nairne, 1990)? We believe that important insight into the composition of memory traces can be gained by studying how component features of the memory trace interact. To investigate this area, we applied the speeded-classification paradigm (Garner, 1974; Garner \& Felfoldy, 1970), extensively used in the study of perceptual interactions, to the study of interactions among components of the short-term memory trace.

In applying speeded-classification techniques to the domain of the memory trace, we have chosen to concentrate on temporal and spatial attributes of stimuli in situations in which both temporal and spatial position can vary. Considerable controversy exists over the nature of temporal and spatial encoding, as well as whether or not these two dimensions of information are independent of each other (see, e.g., Clayton \& Habibi, 1991; Healy, 1982; Mandler \& Anderson, 1971). We address the pos-

We thank Alice Healy, Gregory Jones, Moshe Naveh-Benjamin, and the editor for helpful comments on an earlier version of this paper. Correspondence concerning this article should be addressed to A. Dutta, who is now at the Department of Psychology, Rice University, Box 1892, Houston, TX 77251-1892. sible interdependency by asking whether memory for an item's temporal position can be accessed without regard to its prior spatial position, and whether the recovery of spatial position is influenced by prior temporal position. Assuming that both temporal and spatial attributes of stimuli are encoded, is it possible to attend to one encoded value without experiencing intrusion from the other?

\section{THE SPEEDED-CLASSIFICATION PARADIGM}

The speeded-classification paradigm has been used by perception researchers to determine the nature of perceptual processing (e.g., Garner, 1974; Melara, 1989; Pomerantz, 1983). Within this paradigm, it is possible to assess whether different dimensions of stimuli, such as color saturation and brightness, can be processed independently of each other or whether judgments of one attribute are influenced by variations of the other. Typically, subjects are required to make speeded classifications of stimuli on the basis of variations of a single primary stimulus dimension (e.g., saturation level). Different sessions are conducted in which a second dimension of interest (e.g., brightness) is either held at a constant value, varied orthogonally to the primary dimension, or varied so that it is positively or negatively correlated with the primary dimension. The researcher is interested in determining what effect, if any, irrelevant variation on one of the dimensions has on the classification of the other.

Interaction of the two dimensions is said to occur if subjects are unable to attend selectively to the dimension to be classified. If speeded judgments of saturation are affected by irrelevant variation of brightness, the dimensions of saturation and brightness are said to interact, or to be integral; if the processing of one dimension is unaffected by variations in the other, the dimensions are said 
to be separable. So-called Garner interference, defined as the difference in reaction time (RT) between classifications in which the irrelevant dimension is held constant and classifications in which the irrelevant dimension is varied orthogonally, is the main measure of dimensional interaction. Dimensions that interact yield Garner interference; those that are separable do not.

A more detailed analysis of dimensional interaction is possible through the comparison of conditions in which the correlation between the relevant and to-be-ignored dimensions is varied. Redundancy gain occurs when positive (or negative) correlation leads to performance faster than the baseline condition (in which the value of the irrelevant dimension is held constant). Redundancy loss occurs when positive (or negative) correlation leads to performance slower than baseline. The classification of separable dimensions is typically unaffected by correlational redundancy.

With certain types of interacting dimensions, an effect of dimensional congruity is obtained. Congruity scores are computed by calculating RTs and accuracy for positively and negatively correlated trials within a session in which both types of trials are randomly intermixed (known by convention as the filtering condition). The difference in RT between congruent (positively correlated) and incongruent (negatively correlated) trials defines the congruity score. Congruity scores that differ significantly from zero are indicative of a meaning-based, as opposed to a perceptually integral, relation between the dimensions under study. Such dimensions are called correspondence dimensions because they exhibit some meaningful, often learned, relationship. Melara and Marks (e.g., Melara, 1989; Melara \& Marks, 1990) have documented examples of postperceptual interaction based on obtained patterns of interference between correspondence dimensions. For example, they obtained congruity effects when the dimension pitch was paired with the visually presented words " $\mathrm{HI}$ " and "LO." When these stimulus dimensions corresponded (e.g., high pitch paired with "HI'), RTs were faster than when they did not (e.g., low pitch and "HI").

The speeded-classification paradigm has been used most often to uncover patterns of perceptual crosstalk, but it can be extended to new domains. Melara and Nairne (1991), for example, treated time as a dimension and asked whether past processing of a stimulus necessarily affects judgments made about that stimulus in the present, and whether present stimulus values must affect decisions about the past. In that application, the question of interest asked whether processing interactions would occur between values of the same dimension, but at different points in time. In the present work, our interest is exclusively in the past: Given that multiple features of a presentation episode are encoded, is it possible to attend selectively to one attribute of the memory trace without experiencing intrusions from others?

To illustrate, suppose that a subject is presented at Time $t l$ with a sequence of geometric figures that occur at different spatial positions on a computer screen. At test,
Time $\mathbf{t} 2$, the subject is re-presented with one of the figures and is asked to make a speeded judgment regarding that item's prior spatial position. Presumably, the mnemonic representation of the test figure contains multiple features, including time of occurrence, spatial position, color, and so forth. With the speeded-classification paradigm, it can be determined whether, over trials, the subject can make judgments about spatial position without RTs being affected by concurrent variation of the values of the other features. In the language of the speededclassification paradigm, it can be ascertained whether encoded features of a memory trace must interact (e.g., display Garner interference), or are separable, producing no mutual interference.

The experiments reported here were designed to examine the effect of perceptual variation at the time of encoding on the recovery of specific dimensional information at test. We used temporal order and spatial position as our two mnemonic dimensions. Two geometric shapes occurred either first or second in time and above or below a fixation point. Following a short delay, the subject was required to make judgments about either the temporal or the spatial dimension. Importantly, because the decision probe always followed the offset of the presentation stimuli, correct performance required the subject to remember the specific prior temporal or spatial presentation values. In Experiment 1, subjects were required to classify stimuli according to temporal occurrence, while ignoring irrelevant variation of spatial position. In Experiment 2, spatial position was recalled and temporal occurrence was to be ignored. Experiment 3 required that subjects make a speeded response regarding the temporal dimension, followed by an unspeeded response about the spatial dimension. In Experiment 4, the value of the spatial dimension was recalled as rapidly as possible and an unspeeded response was made regarding the temporal occurrence of the stimulus.

In summary, the speeded-classification paradigm makes it possible to ask questions regarding the nature of dimensional interaction. We extend the paradigm here to the study of encoded dimensional values that reside in the short-term memory trace. The analyses afforded by the use of the speeded-classification paradigm allow us to determine whether or not spatial and temporal information in the memory trace are interrelated. If the mnemonic attributes of temporal and spatial position are stored independently, and if temporal occurrence and spatial $\propto$ currence are separable dimensions, then recall of spatial values should not be affected by the presence of conflicting or consistent temporal information, and vice versa. If the recall of one dimension is influenced by the value on the corresponding dimension, the representation of the two dimensions must not be entirely independent.

\section{EXPERIMENT 1}

In Experiment 1, we examined the influence of variation in spatial position at the time of encoding on the sub- 
sequent recall of temporal order. Because information on a screen or page is usually processed in a top-to-bottom manner (as in reading text), position tends to be correlated with temporal occurrence in that earlier items have spatially higher positions. It is likely, therefore, that spatial and temporal occurrence are correspondence dimensions (Melara \& Marks, 1990) and will exhibit dimensional interaction. In the language of the speeded-classification paradigm, top may be described as positively correlated to first and negatively correlated to second in the stimulus combinations used here. On each of a series of trials, two stimuli, a circle and a square, were presented to the subject. A given stimulus could appear first or second in time and above or below a fixation point. The subject's task was to remember the temporal order of presentation of the stimuli. After a delay of $1 \mathrm{sec}$, one of the stimuli was shown in the center of the screen. The subject then pressed a response key to indicate whether this shape had been shown first or second.

Each subject participated in five conditions. In the baseline conditions, the stimuli always appeared above the fixation point or below the fixation point. In the positively correlated condition, the first stimulus appeared above the fixation point and the second one appeared below. In the negatively correlated condition, the first stimulus appeared below the fixation point and the second one appeared above. Finally, in the filtering condition, positively and negatively correlated trials were intermixed so that temporal and spatial position varied orthogonally.

\section{Method}

Subjects. Twenty Purdue University undergraduate students served as subjects in partial fulfillment of an introductory psychology course requirement. All subjects reported normal color vision.

Apparatus and Stimuli. The stimuli were presented in red on the dark screen of IBM-compatible AT-style personal computers. The color red was chosen to minimize any effect of an afterimage on the screen. The viewing distance was not fixed, but was approximately $50 \mathrm{~cm}$. Two stimuli were used: a circle and a square. The square was $0.8 \mathrm{~cm}$ wide, subtending a visual angle of approximately $0.92^{\circ}$. The circle was $0.9 \mathrm{~cm}$ in diameter, subtending a visual angle of approximately $1.03^{\circ}$. The fixation point was a plus sign measuring $0.3 \mathrm{~cm}$. The stimulus appeared $1.3 \mathrm{~cm}$ above or below fixation, so that the entire stimulus display subtended a visual angle of $4.95^{\circ}$ in height.

With two possible orderings of temporal occurrence (circle first and square second; square first and circle second), two possible spatial orderings (circle above and square below; square above and circle below), and two possible response cues (circle or square), eight different stimulus combinations were created. These stimulus combinations were used to create five experimental blocks of 96 trials each; each stimulus combination within a block occurred equally often and in random order. The stimulus combinations in the baseline blocks were modified to control for one dimension, as described below.

Baseline. The purpose of the baseline conditions was to establish a control condition against which further measures could be compared. In an attempt to control for the spatial-position component of the task, the two shapes employed appeared in the same position on the screen, one following the other. Two baseline conditions were used: both shapes appearing above fixation, and both shapes appearing below fixation. Two stimulus combinations were possible: the square appearing first, followed by the circle, and the circle appearing first, followed by the square. Following the offset of the second stimulus, one of the shapes was shown as the query for the response. Either the circle or the square was cued for response, resulting in four trial types.

Positively correlated. Two stimulus combinations were presented: the square above, appearing first, with the circle below, appearing second; and the circle above, appearing first, with the square below, appearing second. Either the circle or the square was cued for response, resulting in four trial types.

Negatively correlated. Two stimulus combinations were presented: the circle below, appearing first, with the square above, appearing second; and the square below, appearing first, with the circle above, appearing second. Again, either the circle or the square was cued for response, resulting in four trial types.

Filtering. The dimensions of temporal occurrence and spatial position were varied orthogonally so that half the time position and order were positively correlated and half the time position and order were negatively correlated, with presentation order randomized. The eight trial types used in this condition are described above.

Procedure. Responses were made by pressing either the " $V$ " or the " $N$ " key on the bottom row of the computer's keyboard. For half the subjects (selected randomly), the temporal attribute first was assigned to the " $\mathrm{V}$ " key and the temporal attribute second was assigned to the " $\mathrm{N}$ " key; for the remaining subjects, this assignment was reversed. The subjects were instructed to use the appropriate assignment and were provided with cards summarizing the key assignments. In addition, they were told to respond on the basis of temporal occurrence, and to ignore spatial position. They were told that they were participating in an RT experiment and that they should respond as quickly as possible without making too many errors. The subjects were tested individually in rooms containing one computer. The stimulus durations, intervals, and response latency and accuracy were controlled and recorded by the computer.

Each trial began with the presentation of the fixation point for $2 \mathrm{sec}$. This was followed by the presentation of one of the stimuli either above or below fixation, depending on the condition. The onset of the second stimulus always coincided with the offset of the first. Each stimulus shape was displayed for $1 \mathrm{sec}$. The test stimulus was displayed $1 \mathrm{sec}$ after the second stimulus went off the screen. It remained in view until the response was made. If the subject made an error, the computer emitted a tone for $500 \mathrm{msec}$. The start of each trial was separated from the response for the previous trial by an interval of $500 \mathrm{msec}$. Prior to presentation of the experimental blocks, subjects completed a 48-trial practice block made up of baseline trials. The baseline, correlated, and filtering blocks were then presented in a predetermined quasirandom order. Presentation order was counterbalanced across subjects in a Latin square design.

Data analysis. Data from the practice block were not analyzed. RTs under $150 \mathrm{msec}$ or over $2,000 \mathrm{msec}$ were discarded. RT cutoffs in all of the experiments were chosen to eliminate approximately $1 \%$ of the trials, which were considered anticipations or outliers. Error trials were excluded from the RT analyses, but were analyzed separately. The overall error rate was approximately .03 .

\section{Results}

RTs remained fairly constant across conditions (see Table 1), differing by no more than $10 \mathrm{msec}$. An analysis of variance (ANOVA) performed on mean RTs, with condition and response assignment as factors, revealed no effect of condition or assignment $[F<1$ and $F(1,18)=$ $1.26, M S_{\mathrm{e}}=83,844, p>.25$, respectively]; the condition $\times$ assignment interaction also did not reach significance $(F<1)$. Subjects were slightly more accurate in 
Table 1

Mean Response Times (RTs, in Milliseconds) and Error Proportion as a Function of Condition in Experiment 1

\begin{tabular}{|c|c|c|c|c|c|c|c|}
\hline \multicolumn{2}{|c|}{ Baseline } & \multicolumn{2}{|c|}{$\begin{array}{l}\text { Positively } \\
\text { Correlated }\end{array}$} & \multicolumn{2}{|c|}{$\begin{array}{l}\text { Negatively } \\
\text { Correlated }\end{array}$} & \multicolumn{2}{|c|}{ Filtering } \\
\hline RT & Error & RT & Error & RT & Error & RT & Error \\
\hline 545 & .035 & 542 & .035 & 540 & .035 & 550 & .025 \\
\hline
\end{tabular}

the filtering condition [error proportion (EP) $=.025$ vs. .035 in the remaining conditions], but an ANOVA performed on mean accuracy showed no effect of condition $\left[F(4,76)=1.11, M S_{\mathrm{e}}=.00041, p>.35\right] .{ }^{1}$ Finally, additional analyses of the order of block presentation revealed no significant effects on either RTs or accuracy $(F s<1)$.

Congruity scores were computed by subtracting the mean RT for the negatively correlated trials from the mean RT for the positively correlated trials within the filtering condition. The means for positively and negatively correlated trials differed by only $1 \mathrm{msec}(549$ and $550 \mathrm{msec}$, respectively). Neither the congruity scores computed across nor within assignments reached significance $(p>$ .10 for $t$ tests computed on each difference). Thus, the congruity analysis of the filtering condition showed no effect of positive versus negative correlation.

\section{Discussion}

A given stimulus must appear in a particular place and at a particular time. It has been amply demonstrated that these stimulus dimensions are encoded under both intentional (e.g., Bjork \& Healy, 1974; Lee \& Estes, 1977) and incidental (Mandler, Seegmiller, \& Day, 1977; Park \& Mason, 1982) learning conditions. Here, the relatively stable mean RTs across conditions suggested that information regarding temporal occurrence could be accessed independently of spatial position information. Under none of the presentation conditions did the spatial position of the stimulus affect the response to temporal occurrence. From the perspective of the speeded-classification paradigm, this would be the case if these two attributes of the stimuli were represented in the memory trace as separable dimensions.

This outcome has bearing on the literature regarding temporal information processing. The results suggest that the retention or retrieval of temporal information is separable from that of spatial position information. Previous work (e.g., Healy, 1982) on short-term memory has shown temporal and spatial position information to be linked intimately in the form of a temporal-spatial code. Such results, however, may reflect strategic processing by the subject in an effort to maximize immediate memory performance. In speeded classification, it is often in the subject's interest to ignore one dimension while processing another; indeed, in the present case, we instructed our subjects to ignore the irrelevant spatial dimension. Thus, the linking of temporal and spatial information may not be an obligatory process, but rather a strategic one that is employed only under restricted conditions. The present results show that it is possible for subjects to attend selectively to the temporal dimension, suggesting separable components of the memory trace.

\section{EXPERIMENT 2}

Experiment 1 demonstrated that the recall of temporal order was unaffected by variation in the spatial position of the stimuli at encoding. In Experiment 2, we evaluated whether the obtained pattern of separability of spatial and temporal occurrence is symmetrical. That is, can spatial location be accessed equally well with or without variation in temporal occurrence? In Experiment 2, the subjects were told to attend and respond to the spatial location of the stimulus, ignoring temporal occurrence. The stimuli, procedure, and data analysis were identical to those in Experiment 1, except for the presentation of the baseline condition, which is described below. With spatial position as the primary dimension, it was also possible to evaluate any dependency of spatial encoding on temporal encoding. Once again, the nature of the relation between temporal and spatial information is particularly interesting in light of obtained evidence that spatial information is sometimes recoded into temporal-spatial patterns (e.g., Healy, 1977, 1982) or otherwise depends on temporal associations (e.g., Clayton \& Habibi, 1991). Past evidence of an influence of temporal order on the recall of spatial position suggests that the pattern of separability obtained in Experiment 1 may not be found when spatial position is the primary dimension.

\section{Method}

Subjects. Twenty new subjects from the same population as in Experiment 1 were tested. The subjects were randomly assigned to either the above-to- $\mathrm{V}$ and below-to- $\mathrm{N}$ or the below-to- $\mathrm{V}$ and above-to- $\mathrm{N}$ response assignment, with the constraint that each assignment was used equally often.

Apparatus and Stimuli. The apparatus and stimuli were identical to those in Experiment 1, except for the baseline condition. To control for temporal occurrence in the baseline condition, the two stimulus shapes appeared simultaneously, one above fixation and the other below. As in Experiment 1, the target shape appeared in the center of the screen as the query for response. In the baseline condition, possible stimulus combinations were: circle above with square below, and square above with circle below.

Procedure. The procedure was identical to that in Experiment 1, except that the subjects responded on the basis of spatial position.

Data analysis. Data from the practice block were discarded, as were RTs under $150 \mathrm{msec}$ or over $2,000 \mathrm{msec}$. Error trials were excluded from the RT analyses, but were analyzed separately. The overall error rate was .04 .

\section{Results}

As can be seen in Table 2, there was little variation in mean RTs, with the greatest difference being $12 \mathrm{msec}$. An ANOVA performed on mean RTs with condition and response assignment as factors revealed no effect of condition or assignment $(F \mathrm{~s}<1)$. The condition $\times$ assignment interaction also did not reach significance $(F<1)$. An ANOVA performed on mean accuracy revealed no 
Table 2

Mean Response Times (RTs, in Milliseconds) and Error Proportion as a Function of Condition in Experiment 2

\begin{tabular}{|c|c|c|c|c|c|c|c|}
\hline \multicolumn{2}{|c|}{ Baseline } & \multicolumn{2}{|c|}{$\begin{array}{l}\text { Positively } \\
\text { Correlated }\end{array}$} & \multicolumn{2}{|c|}{$\begin{array}{l}\text { Negatively } \\
\text { Correlated }\end{array}$} & \multicolumn{2}{|c|}{ Filtering } \\
\hline RT & Error & RT & Error & RT & $\overline{\text { Error }}$ & RT & Error \\
\hline 582 & .045 & 584 & .04 & 594 & .045 & 588 & .03 \\
\hline
\end{tabular}

effect of condition $\left[F(3,57)=2.10, M S_{\mathrm{e}}=.0005, p>\right.$ $.10]$, although the subjects were slightly more accurate in the filtering condition. Additionally, the congruity analysis of the filtering condition showed no effect of positive versus negative correlation $(t \mathrm{~s}<1)$. Mean RT for both the positively and negatively correlated trials was $588 \mathrm{msec}$. As in Experiment 1, no reliable effects of block counterbalancing were obtained for either the RT or the accuracy measures.

\section{Discussion}

The results of Experiment 2 were in agreement with those of Experiment 1: There was no evidence of interaction of spatial and temporal information in the shortterm memory trace. The lack of an effect of temporal variation on the recall of spatial attributes is consistent with the pattern of separability found in Experiment 1, but conflicts somewhat with previous findings demonstrating the encoding of temporal-spatial patterns in immediate memory environments. However, whereas Healy $(1975,1982)$ reported evidence of temporal-spatial pattern encoding, Nairne and Dutta (1992; see also Anderson, 1976) found no evidence that such patterns were used in a (long-term) incidental memory task. Taken together, the two experiments reported here support a hypothesis of dimensional separability of space and time. These results, considered together with previous work, suggest that coding strategies may be task dependent. Subjects appear able to ignore temporal information when that information is of no use (i.e., is uncorrelated with spatial attributes) or may hurt spatial recall.

\section{EXPERIMENT 3}

In Experiment 1, the subjects recalled the temporal occurrence of a stimulus equally rapidly, whether or not temporal and spatial position corresponded. This result suggested that temporal and spatial information could be accessed independently from the memory trace. However, because that experiment demanded the recall of only the temporal dimension, and the subjects were told to ignore spatial position, it is conceivable that the spatial position of the stimulus was never encoded. It is possible, then, that subjects selectively ignored variation on the irrelevant dimension at the time of presentation and that this information never became part of the memory trace. If this was the case, we tested the perceptual separability of space and time-not their separability in the memory trace. Alternatively, spatial position may have been encoded, but not actively maintained in short-term memory.
Accordingly, to ensure that subjects actively attended to both the temporal and spatial features of stimulus occurrence, Experiment 3 required that subjects remember both when and where a given stimulus occurred. The design of the stimuli and conditions was the same as in Experiment 1, but after the response regarding temporal occurrence was made, the subjects made an unspeeded response about the spatial position of a test stimulus. Because both the spatial and the temporal dimensions had to be encoded and kept current in short-term memory, any pattern of interactivity or separability found in this experiment presumably can be attributed to the representation and access of these dimensions in the memory trace.

\section{Method}

Subjects. Thirty new subjects from the same population as before were tested.

Apparatus and Stimuli. The apparatus and stimuli were identical to those in Experiment 1. Because two independent responses were required, there were 16 possible stimuli. All stimuli within a block were used equally often and were presented in random order.

Procedure. As before, two response assignments were used. In one, first and above were assigned to the " $V$ " key, and second and below to the " $N$ " key. In the other, first and above were assigned to the " $\mathrm{N}$ " key, and second and below to the " $\mathrm{V}$ " key. An equal number of subjects used each assignment. There was no separate practice block; instead, the first 16 trials of each block were counted as practice, leaving 80 trials for analysis in each condition. It was decided to include practice trials in each block, because the second response in the baseline condition was not meaningfully related to the task (we discuss this point further below). The trial sequence was the same as in the previous experiments, except that after a response was made on the primary dimension (temporal occurrence), either the same shape or the alternate shape was shown and a response had to be made regarding spatial position. Error feedback, if any, followed each response. All possible trial types were constructed and all types were used equally often.

Data analysis. Data from the 16 practice trials at the beginning of each block were discarded, as were RTs under $150 \mathrm{msec}$ or over $2,500 \mathrm{msec}$. RT analyses were performed on the first response only, whereas the accuracy of both responses was analyzed. Trials on which an error was made on either response were excluded from RT analyses. Approximately $6 \%$ of the trials were excluded for this reason. The overall error rate was .033 .

\section{Results}

Overall RTs were slower in Experiment 3, presumably because subjects were required to make two responses rather than one. Of main interest was that the subjects in this experiment responded more slowly in the negatively correlated and filtering conditions (see Table 3), showing an average increase of $214 \mathrm{msec}$ over the baseline

\section{Table 3}

Mean Response Times (RTs, in Milliseconds) and Error Proportion as a Function of Condition in Experiment 3

\begin{tabular}{|c|c|c|c|c|c|c|c|c|c|c|c|}
\hline \multirow[b]{3}{*}{ RT } & \multicolumn{2}{|c|}{ aseline } & \multicolumn{3}{|c|}{$\begin{array}{l}\text { Positively } \\
\text { Correlated }\end{array}$} & \multicolumn{3}{|c|}{$\begin{array}{l}\text { Negatively } \\
\text { Correlated }\end{array}$} & \multicolumn{3}{|c|}{ Filtering } \\
\hline & \multicolumn{2}{|c|}{ Error } & \multicolumn{3}{|c|}{ Error } & \multirow[b]{2}{*}{ RT } & \multicolumn{2}{|c|}{ Error } & \multirow[b]{2}{*}{ RT } & \multicolumn{2}{|c|}{ Error } \\
\hline & $R_{1}$ & $\mathbf{R}_{\mathbf{2}}$ & RT & $R_{1}$ & $\overline{R_{2}}$ & & $R_{1}$ & $\mathbf{R}_{2}$ & & $R_{1}$ & $\overline{\mathbf{R}_{2}}$ \\
\hline 720 & .03 & 0 & 720 & .02 & .02 & 951 & .03 & .05 & 917 & .03 & .05 \\
\hline
\end{tabular}

Note-RT is temporal; $R_{1}$, temporal; $R_{2}$, spatial. 
and positively correlated conditions. An ANOVA performed on mean RTs showed a significant effect of condition $\left[F(4,112)=30.84, M S_{\mathrm{e}}=13,527, p<.001\right]$. Neither the main effect of assignment nor the assignment $x$ condition interaction reached significance $\left(F_{\mathrm{S}}<1\right)$. In addition, there were some significant, but not easily interpretable, block order effects. However, in all five counterbalancing conditions, the baseline and positively correlated conditions were faster than the negatively correlated and filtering conditions.

ANOVAs also were performed on the mean accuracy of the first and second responses, with condition as a factor. The effect of condition on the the accuracy of the first response was not significant $\left[F(4,116)=1.33, M S_{\mathrm{e}}=\right.$ $.00039, p>.25$ ]. There was, however, a significant effect of condition on the second response $[F(4,116)=$ $\left.16.78, M S_{\mathrm{e}}=.00075, p<.001\right]$, reflecting lower accuracy in the negatively correlated and filtering conditions (EP $=.05$ vs. .02 in the positively correlated condition).

A Ryan-Einot-Gabriel-Welsh Multiple Range test (REGWQ; SAS Institute, 1990) was performed on condition RT means. This test, which controls the Type I experimentwise error rate, revealed that the two baseline condition means and the positively correlated condition mean did not significantly differ, but that all three of these mean RTs were significantly lower than the negatively correlated and filtering condition means $[\alpha(145)=.05$, $\left.M S_{\mathrm{e}}=60,647\right]$.

Within the filtering condition, mean RT for the positively correlated trials was $882 \mathrm{msec}$ and for the negatively correlated trials was $954 \mathrm{msec}$, resulting in a 72msec difference. This effect of congruity computed for the filtering condition was significant both for RTs $\left[F(1,29)=9.01, M S_{\mathrm{e}}=8,658, p<.01\right]$ and for accuracy $\left[F(1,29)=11.52, M S_{\mathrm{e}}=.00079, p<.005\right.$, and $F(1,29)=9.57, M S_{\mathrm{e}}=.00239, p<.005$, for the first and second responses, respectively]. Responding was more accurate when the trials were positively correlated than when they were negatively correlated (EP $=.02$ vs. .05 , respectively). There were no significant block presentation order effects on accuracy.

\section{Discussion}

The major conclusion that can be drawn from this experiment is that spatial variation does affect temporal recall when both dimensions must be actively remembered. In terms of both speed and accuracy, performance was better when the temporal and spatial values were congruent (i.e., positively correlated) than when they were incongruent (i.e., negatively correlated). This resultcoupled with the outcome of Experiment 1, in which no effect of spatial variation was obtained-suggests that subjects performed the task differently in the two experiments.

The empirical pattern of interference, as well as the conditions under which it was obtained, suggest that temporal information in the memory trace is sometimes subject to obligatory interference or crosstalk from spatial informa- tion. The subjects were unable to ignore the spatial value when making a temporal decision, despite the fact that it was in their interest to do so. It may be the fact that temporal and spatial location are meaningfully related that allows the interference to occur. As an example, consider recalling an item from a presented page of text. We naturally think that an earlier item would be presented nearer the top of the page and a later item nearer the bottom. It is possible that it is the conflicting nature of what is conveyed by a "higher position" or an "earlier occurrence" that causes interference in this task.

\section{EXPERIMENT 4}

In Experiment 3, contrary to Experiment 1, evidence for interaction of spatial and temporal information was found. Experiment 4 followed the procedure used in Experiment 3 to test whether spatial information can be accessed independently of temporal information when both dimensions are present and active in the memory trace. This manipulation allowed us to determine whether the pattern of interference found in Experiment 3 was symmetrical, as would be expected if it were due to conflicts in the meaning conveyed by position and time of occurrence.

\section{Method}

Subjects. Thirty-two Purdue undergraduate students participated as before, with equal numbers of subjects using each response assignment. An additional 9 subjects were tested but were excluded because their error rates exceeded 20 . However, the excluded subjects showed the same ordering of RTs across conditions as reported in the results section. Most of these subjects (7 out of 9) received the filtering condition first and it was the extremely high error rate in this condition that led to their exclusion.

Stimuli and Apparatus. The stimuli were identical to those used in Experiment 2, with the addition of a second test stimulus as in Experiment 3

Procedure. The procedure used in Experiment 3 was followed, except that a speeded response to spatial position was followed by an unspeeded response to temporal occurrence.

Data analysis. Data from the 16 practice trials at the beginning of each block were discarded, as were RTs under $150 \mathrm{msec}$ or over $2,500 \mathrm{msec}$. Trials on which an error was made on either response (approximately $7.5 \%$ of the trials) were excluded from RT analyses, but were analyzed separately. The overall error rate was .04 .

\section{Results}

Responding in the negatively correlated condition was $281 \mathrm{msec}$ slower than in the baseline condition. In the filtering condition, responding was $221 \mathrm{msec}$ slower than in the baseline condition (see Table 4). An ANOVA performed on mean RT for the first response with condition and assignment as factors showed that the main effect of condition was significant $\left[F(3,90)=24.03, M S_{\mathrm{e}}=\right.$ $21,891, p<.001]$, but the main effect of assignment and the condition $\times$ assignment interaction were not $(F \mathrm{~s}<1)$. There were no significant block order effects in the RT data. ANOVAs performed on the mean accuracy of the first and second responses showed no effect of condition $\left[F<1\right.$ and $F(3,93)=1.92, M S_{\mathrm{e}}=.0041, p>.10$, 
Table 4

Mean Response Times (RTs, in Milliseconds) and Error Proportion as a Function of Condition in Experiment 4

\begin{tabular}{|c|c|c|c|c|c|c|c|c|c|c|c|}
\hline \multicolumn{3}{|c|}{ Baseline } & \multicolumn{3}{|c|}{$\begin{array}{l}\text { Positively } \\
\text { Correlated }\end{array}$} & \multicolumn{3}{|c|}{$\begin{array}{l}\text { Negatively } \\
\text { Correlated }\end{array}$} & \multicolumn{3}{|c|}{ Filtering } \\
\hline \multirow[b]{2}{*}{ RT } & \multicolumn{2}{|c|}{ Error } & \multirow[b]{2}{*}{ RT } & \multicolumn{2}{|c|}{ Error } & \multirow[b]{2}{*}{ RT } & \multicolumn{2}{|c|}{ Error } & \multirow[b]{2}{*}{ RT } & \multicolumn{2}{|c|}{ Error } \\
\hline & $\mathbf{R}_{\mathbf{1}}$ & $\mathbf{R}_{\mathbf{2}}$ & & $\mathrm{R}_{\mathbf{1}}$ & $\mathbf{R}_{2}$ & & $\mathbf{R}_{1}$ & $\mathbf{R}_{\mathbf{2}}$ & & $\mathbf{R}_{\mathbf{I}}$ & $\mathbf{R}_{\mathbf{2}}$ \\
\hline & .04 & 0 & 636 & .03 & .02 & 836 & .04 & .04 & 776 & .03 & .05 \\
\hline
\end{tabular}

Note $-\mathbf{R T}$ is temporal; $\mathbf{R}_{1}$, temporal; $\mathbf{R}_{\mathbf{2}}$, spatial.

respectively]. Accuracy was slightly lower in the negatively correlated and filtering conditions (averaged EP for the positively correlated and baseline conditions was .03; for the negatively correlated and filtering conditions, the averaged EP was .04). There were no significant block order effects in the accuracy data.

A REGWQ test (SAS Institute, 1990) was performed on condition means. This test revealed that the baseline and positively correlated condition means did not significantly differ but that the negatively correlated and filtering condition means were significantly slower than these means $\left[\alpha(124)=.05, M S_{\mathrm{e}}=57,551\right]$. Moreover, the congruity effect computed for the filtering condition was significant for both RT $\left[F(1,31)=17.30, M S_{\mathrm{e}}=5,873\right.$, $p<.0002]$ and accuracy $\left[F(1,31)=10.14, M S_{\mathrm{e}}=\right.$ $.00130, p<.005$, and $F(1,31)=12.36, M S_{\mathrm{e}}=.00163$, $p<.005$, for the first and second responses, respectively]. Mean RT for the positively correlated trials was $738 \mathrm{msec}$ and for the negatively correlated trials was $817 \mathrm{msec}$, a 79-msec difference. Responding was more accurate when the trials were positively correlated (EP = .02 vs. .04 and .03 vs. .06 for the first and second responses, respectively).

Finally, because the baseline condition involved the simultaneous presentation of both stimuli, one might argue that subjects were faster in this condition because there is a shorter delay between presentation and probe (i.e., $1 \mathrm{sec}$ for both stimuli in baseline). If a shorter delay per se led to better performance, we would expect RTs to have been faster and accuracy to be higher for the second presented stimulus in the nonbaseline conditions. However, this trend was not evident in post hoc analyses of the data with stimulus presentation order and condition as factors.

\section{Discussion}

In contrast to Experiments 1 and 2, both Experiments 3 and 4 showed a significant effect of condition. Subjects were slower to respond in the negatively correlated and filtering conditions. In short, interference of an irrelevant dimension was apparent in Experiments 3 and 4. When both dimensions were encoded, or were maintained actively in memory, it took longer to respond to one dimension in the presence of a conflicting value on the other. Again, the obtained pattern of interference suggested semantic crosstalk.

\section{GENERAL DISCUSSION}

In this study, we explored the relationship of temporal and spatial attributes of the memory trace. In particular, the research directly addressed how the encoding and recall of spatial position was influenced by variation in temporal order and vice versa. Temporal occurrence and spatial position were treated as ordered stimulus dimensions. We did not ask about the nature of processing at designated points in time; rather, we were concerned with the encoding of particular values of temporal occurrence that were to be retrieved only after the stimuli had been processed. Similarly, in the spatial dimension, we were not concerned with the effect of spatial location on current processing (as in studies of spatial stimulus-response compatibility; see Proctor \& Reeve, 1990); rather, we were interested in the retrieval of previously coded spatial location information. The speeded-classification paradigm (Garner, 1974) was used to assess dependencies of spatial and temporal information in the memory trace. Thus, we were able to determine the abilities of subjects to recall only spatial or temporal information while ignoring the alternate dimension.

In Experiments 1 and 2, subjects responded relatively consistently across conditions in which the relation of temporal and spatial information was varied. There was no evidence for any dimensional interaction. It was suggested that this could be due to the separability of the spatial and temporal dimensions at encoding: The subjects had no trouble actively maintaining one physical dimension in short-term memory while selectively ignoring the other. A different pattern of results emerged in Experiments 3 and 4 . When subjects were required to recall the values of the stimuli on both dimensions, irrelevant variation of the alternate dimension produced significant interference.

The pattern of results found in Experiments 3 and 4 closely resembles what Melara and Marks (1990) call semantic crosstalk. As discussed earlier, hallmarks of semantic crosstalk are redundancy gain only in the positively correlated condition, significant Garner interference (i.e., impaired performance in the filtering condition), and an effect of congruity. The most striking aspect of the data in Experiments 3 and 4 was strong Garner interference, coupled with a considerable congruity effect. There was, however, no facilitation in the positively correlated condition relative to the baseline condition (in fact, RTs were slower in this condition when the primary response was to spatial position). The lack of facilitation may have been due to the increased difficulty of remembering two values instead of one (recall that in the baseline condition only one dimension was varied; thus, the second response was always the same). Because of this difference in the amount of information to be recalled, it may not be possible to measure facilitation in comparison with the baseline condition in the latter experiments. The best indicator of interaction between these dimensions may thus be the differ- 
ence between the positively and negatively correlated trials.

Relying only on the findings of Garner interference and congruity effects, a hypothesis of semantic crosstalk between corresponding dimensions is tenable. Consistent with the finding that interference was obtained only when it was not possible for subjects to ignore the irrelevant dimension at encoding, semantic crosstalk is assumed to take place at a postperceptual stage. In this manner, semantic crosstalk corresponds to conceptually (as opposed to data-) driven processing. This places the interference at a postperceptual stage and suggests that the codes for the representation of spatial and temporal information interact in the memory trace, at least under conditions in which both need to be maintained actively to ensure correct responding.

The results of this study can be brought to bear on the question of the automaticity of temporal (or spatial) encoding. In the past, the robustness of temporal encoding has led many researchers to argue that temporal information is encoded automatically (i.e., performed without conscious attentional control; see, e.g., Hasher \& Zacks, 1979, but see Zacks, Hasher, Alba, Sanft, \& Rose, 1984, for contradictory evidence). Similarly, there has been debate over whether the encoding of spatial information may best be described as occurring automatically, or under the intention or control of the subject (Hasher \& Zacks, 1979; Mandler et al., 1977; Naveh-Benjamin, 1987). The results of the first two as opposed to the second two experiments in this paper suggest that temporal and spatial influences on recall clearly depend on the particular task demands. This in turn suggests that temporal and spatial information are not always accessed from the memory trace. Alternatively, it is possible that in the first two experiments the irrelevant dimension was either never encoded or simply not actively maintained.

To test the possibility that encoding occurs even when subjects are instructed to ignore the irrelevant dimension, we tested additional subjects in the temporal and spatial filtering tasks used in Experiments 1 and 2. A given subject participated in either the temporal or spatial condition. Following 96 trials in which memory for only the temporal (or spatial) attribute was tested, an additional trial was presented in which the subject also had to respond to the spatial position (or temporal occurrence) of one of the stimuli. The subjects were not told of this extra test trial beforehand; rather, they were instructed to ignore the irrelevant dimension. Twenty-eight subjects were tested in each of the two filtering conditions: temporal and spatial. Percent correct and congruity scores were computed for each group (see Table 5).

The subjects in the temporal filtering group performed at about chance accuracy on the spatial test $(53.4 \%$ correct; chance would be $50 \%$ ). Also, this group showed little evidence of a congruity effect [positively correlated trial mean RT $=641 \mathrm{msec}$; negatively correlated trial mean $\mathrm{RT}=654 \mathrm{msec} ; F(1,27)=1.24, M S_{\mathrm{e}}=1,692, p>$ $.25]$. Thus, it appears that subjects neither encoded nor maintained spatial position information in this task.
Table 5

Congruity Scores (in Milliseconds) and Percentage Correct for Temporal or Spatial Recall of an Irrelevant Dimension

\begin{tabular}{lcc}
\hline & \multicolumn{2}{c}{ Relevant Dimension } \\
\cline { 2 - 3 } & Temporal & Spatial \\
\hline $\begin{array}{l}\text { Congruity score } \\
\text { \% Correct on } \\
\text { irrelevant dimension }\end{array}$ & 13 & 10 \\
\hline
\end{tabular}

The spatial filtering group showed better performance on the temporal test, with $67.5 \%$ correct responses. Still, this group showed little sign of a congruity effect [positively correlated trial mean RT $=729 \mathrm{msec}$; negatively correlated trial mean RT $=739 \mathrm{msec}(F<1)$ ]. Why was this group able to perform above chance on the test trial while suffering no interference from the irrelevant temporal dimension? Subjects may have been able to retrieve temporal position information from the trace used to encode spatial position. It is possible that some subjects stored the stimulus information in a verbal form, remembering "circle" and "square" and their respective positions as they were shown. If, for example, the square was both first in time and below fixation, so that the circle was both second and above fixation, the subject could rehearse something like, "square below, circle above." Note that temporal order information is retained in this sort of encoding - the square was shown first and is rehearsed first. Although there is much evidence that spatial information can be retained in a nonverbal, analogical form (e.g., Healy, 1982), some subjects may have adopted a verbal encoding strategy. Presumably, because the temporal information is never accessed (except at the final test trial), it produces no conflicting semantic or response information, allowing performance to proceed without an effect of congruity.

We have tried to show that the speeded-classification paradigm can bring additional insight to questions regarding the mandatory or optional processing of remembered stimulus attributes. The extension of this paradigm to memory research may provide new or additional support for explanations of problems regarding the nature of the memory trace. It will be particularly interesting to apply these techniques to experimental contexts in which the memory load is more demanding. In the present experiments, although operationally we were tapping the shortterm memory trace, the memory load was minimal. Traditionally, short-term memory experiments have been done with larger memory loads (three or more items) and retention has been assessed after varying periods of distraction (e.g., Healy, 1975). It remains to be seen whether the patterns of interference in the present experiments will hold under these more difficult and effortful retrieval conditions.

\section{REFERENCES}

ANDERSON, R. E. (1976). Short-term retention of the where and when of pictures and words. Journal of Experimental Psychology: General, $105,378-402$. 
Buork, E. L., \& HeALY, A. F. (1974). Short-term order and item retention. Journal of Verbal Learning \& Verbal Behavior, 13, 80-97.

Bower, G. H. (1967). A multicomponent theory of the memory trace. In K. W. Spence \& J. T. Spence (Eds.), The psychology of learning and motivation (Vol. 1, pp. 229-325). New York: Academic Press.

Clayton, K., \& HaBiBi, A. (1991). Contribution of temporal contiguity to the spatial priming effect. Journal of Experimental Psychology: Learning, Memory, \& Cognition, 17, 263-271.

GARNER, W. R. (1974). The processing of information and structure. Potomac, MD: Erlbaum.

Garner, W. R., \& Felfoldy, G. L. (1970). Integrality of stimulus dimensions in various types of information processing. Cognitive Psychology, 1, 225-241.

HASher, L., \& Zacks, R. T. (1979). Automatic and effortful processes in memory. Journal of Experimental Psychology: General, 108, 356-388.

HeALY, A. F. (1975). Coding of temporal-spatial patterns in short-term memory. Joumal of Verbal Learning \& Verbal Behavior, 14, 481-495.

Healy, A. F. (1977). Pattern coding of spatial order information in short-term memory. Journal of Verbal Learning \& Verbal Behavior, 16, 419-437.

Healy, A. F. (1982). Short-term memory for order information. In G. H. Bower (Ed.), The psychology of learning and motivation (Vol. 16, pp. 191-238). New York: Academic Press.

JoNEs, G. V. (1976). A fragmentation hypothesis of memory: Cued recall of pictures and of sequential position. Joumal of Experimental Psychology: General, 105, 277-293.

LEE, C. L., \& EsTES, W. K. (1977). Order and position in primary memory for letter strings. Journal of Verbal Leaming \& Verbal Behavior, 16, 395-418

Mandler, G., \& Anderson, R. E. (1971). Temporal and spatial cues in seriation. Journal of Experimental Psychology, 90, 128-135.

Mandler, J. M., Seegmiller, D., \& Day, J. (1977). On the coding of spatial information. Memory \& Cognition, 5, 10-16.

MElaRA, R. D. (1989). Dimensional interaction between color and pitch. Journal of Experimental Psychology: Human Perception \& Performance, 15, 69-79.

Melara, R. D., \& Marks, L. E. (1990). Perceptual primacy of dimensions: Support for a model of dimensional interaction. Journal of Experimental Psychology: Human Perception \& Performance, 16, 398-414.
Melara, R. D., \& NaIRNe, J. S. (1991). On the nature of interactions between the past and the present. Journal of Experimental Psychology: Learning, Memory, \& Cognition, 17, 1124-1135.

NaIRNe, J. S. (1990). A feature model of immediate memory. Memory \& Cognition, 18, 251-269.

NaIRne, J. S., \& DutTA, A. (1992). Spatial and temporal uncertainty in long-term memory. Journal of Memory \& Language, 31, 396-407.

Naveh-BENJAMIN, M. (1987). Coding of spatial location information: An automatic process? Journal of Experimental Psychology: Learning, Memory, \& Cognition, 13, 595-605.

PARK, D. C., \& MASON, D. A. (1982). Is there evidence for automatic processing of spatial and color attributes present in pictures and words? Memory \& Cognition, 10, 76-81.

Pomerantz, J. R. (1983). Global and local precedence: Selective attention in form and motion perception. Journal of Experimental Psychology: General, 112, 515-540.

Proctor, R. W., \& ReEve, T. G. (1990). Stimulus-response compatibility: An integrated perspective. Amsterdam: North-Holland.

SAS INSTITUTE (1990). SAS/STAT user's guide: Version 6 (4th ed., Vol. 1). Cary, NC: SAS Institute.

Tulving, E., \& Bower, G. H. (1974). The logic of memory representations. In G. H. Bower (Ed.), The psychology of learning and motivation (Vol. 8, pp. 265-301). New York: Academic Press.

Tulving, E., \& Watkins, M. J. (1975). Structure of memory traces. Psychological Review, 82, 261-275.

Zacks, R. T., Hasher, L., Alba, J. W., Sanft, H., \& Rose, K. C (1984). Is temporal order encoded automatically? Memory \& Cognition, 12, 387-394.

\section{NOTE}

1. Because there were no reliable differences between response assignments or baseline conditions, these values are combined in the tables. For clarity of description, assignment is not reported as a factor in the accuracy analyses.

(Manuscript received June 29, 1992; revision accepted for publication November $20,1992$. 